\title{
Power-Adjusted Random Access to a Wireless Channel
}

\author{
Young-June Choi and Kang G. Shin \\ The University of Michigan, Ann Arbor, MI 48109-2121, U.S.A. \\ Email: \{yjunchoi, kgshin\}@eecs.umich.edu
}

\begin{abstract}
The operation of widely-deployed random access to wireless networks is based on limited information on the result of each access attempt. When making a random access attempt, users usually do not know the exact amount of transmit power to make it successful. Also, upon failure of a transmission attempt, a user cannot tell whether the failure was caused by collision with other simultaneously-transmitting users or by his use of insufficient transmit power. To handle lack of information on the cause of failure, we propose an innovative Cause-of-Failure $(\mathrm{CoF})$ resolution which increases the transmit power after a given number of consecutive unsuccessful access attempts when the probability that a given failure is caused by collision becomes sufficiently low. To exploit the thus-achieved transmit power for the next random access attempt, we also determine the Causeof-Success (CoS) based on the number of consecutive successful attempts, i.e., whether to decrease or maintain the present transmit power probabilistically. This way, users can adjust their transmit power for random access, which we call Auto Power Fallback (APF). We evaluate APF by modeling analysis and numerical computation based on the slotted Aloha, showing that APF makes significant energy-savings for uplink random accesses while achieving good performance.
\end{abstract}

\section{INTRODUCTION}

Aloha-type random access mechanisms have been implemented mainly for uplink communications in cellular networks. Despite its wide use, it is practically impossible for a user to acquire the perfect channel information for a successful transmission, and hence, a random access is attempted with only partial information about the channel before using it.

For these reasons, it is important to devise a poweradjustment scheme for channel-condition-unaware random access systems. The well-known problem in random access is the difficulty in distinguishing a collision from the failure caused by a low transmit power. Increasing the transmit power blindly to combat the failure will waste energy without benefit. Hence, determining a suitable transmit power to use is essential for energy savings, a critical issue in all wireless mobile systems [1]. An extreme solution for power management is to always use full transmit power. Then, no access failure will occur due to use of insufficient transmit power within the given transmission range. This scheme, however, does not only fail to meet the requirement of energy-savings, but also cause unfairness in terms of uneven successful access probabilities because of the capture effect [2], [3].

In the traditional approach to random access, a collision occurs when multiple users attempt to access a channel simultaneously. However, by the virtue of capture effect, a user can succeed in his transmission while the others' transmissions fail if a receive power from that user is larger than the sum of others' by more than a given threshold. So, in using full transmit power, the user who is located near the AP will succeed in his transmission attempt with a higher probability than the others farther away from the AP [9]. This is the wellknown near-far effect.

To overcome the problem of power assignment, CDMA systems have already deployed a power-ramping algorithm [4]. The power-ramping algorithm increases the transmit power by one step on failure of a random access attempt, while using the slotted Aloha. Following the algorithm in general random access systems, the transmit power would be increased even in case of collision-induced failures. It wastes energy by using excessive transmit power that also interferes with other users. Moreover, this algorithm becomes inefficient if the power ramping always starts from the initial transmit power level, although it can reuse the latest-used power level.

To remedy the above problems, we propose a random access solution that maintains a proper transmit power level. The conventional random access approach has been to find a collision resolution method based on random backoffs. We develop a new concept of failure resolution that seeks an adequate transmit power as well as collision resolution. We tackle this problem with a probabilistic approach and devise a Cause-of-Failure ( $\mathrm{CoF}$ ) estimation algorithm that estimates whether the failure is caused by a collision or by use of insufficient transmit power. Next, to reuse the thus-achieved transmit power level for the next random access attempt, we also propose a Cause-of-Success (CoS) estimation algorithm that estimates whether or not to decrease the current transmit power before attempting a random access.

Traditionally, exploiting the capture effect for slotted Aloha has been regarded helpful in enhancing the throughput of pure slotted Aloha. For this, the transmit power was randomized [5]-[8], and especially the authors of [8] investigated power control for slotted Aloha to improve system capacity. But their underlying assumption was that power variations due to distance are eliminated by perfect power control. The performance of Aloha under the near-far effect is addressed in [9], and its stability studied in [10] by adaptively varying the probability of retransmission. In [11], a tree-splitting collision resolution protocol is proposed, which operates according to residual battery energy. The authors of [12] studied a collision model for CDMA systems and referenced many power control methods. To our best knowledge, little has been done on resolving random-access failures by considering both collision resolution and transmit power adjustment together.

The remainder of this paper is organized as follows. Section II describes our system model and assumptions, and Section III proposes algorithms that consider both transmit power 
management and collision resolution together. Section IV analyzes the performance of the algorithms by modeling it with a discrete-time Markov chain, and Section V evaluates them numerically and using simulation. The paper concludes with Section VI.

\section{SyStem MOdel AND Assumptions}

\section{A. Assumptions}

We consider a wireless network that consists of multiple cells or clusters, each of which is composed of an AP and many users (or wireless terminals). As is commonly the case, random access is used for uplink communications from users to the AP. The air medium is assumed to be accessed randomly by following the slotted Aloha. A packet arriving (generated) during time slot $i$ is transmitted at time slot $i+1$. By the nature of random access, users are informed of the result of a transmission attempt only when it is successful. If the attempt was unsuccessful, there is usually no explicit response from the receiver, so users estimate the failure from the absence of $\mathrm{ACK}$ or the subsequent procedure (e.g., channel allocation) over the downlink. For tractability of our analysis, as in the literature (see, for example, [13]), a user terminal is assumed to learn whether or not an access attempt was successful at the end of each slot from the AP's feedback.

Throughout this paper, we use $G$ to denote the total effective offered load. Since Aloha-type random access methods use random backoffs for their collision resolution, the total offered load includes new and backlogged arrivals. The number of packets generated in the network follows a Poisson distribution with mean rate $G$.

A certain user's random access attempt can be viewed successful due to the capture effect even if other users made simultaneous attempts. That is, when the receive power from a user $k$ is larger than the sum of other users' and background noise $\mathcal{N}$, which is equivalent to the receive signal-to-noise ratio (SNR), $\Gamma$, by a given threshold $\alpha$, user $k$ 's packet can be captured by the receiver. Let $\mathcal{R}_{k}$ be the AP's receive power of user $k$ 's transmission, then user $k$ 's packet will be captured if

$$
\Gamma_{k}=\frac{\mathcal{R}_{k}}{\sum_{i \neq k} \mathcal{R}_{i}+\mathcal{N}} \geq \alpha,
$$

where the threshold $\alpha$ is a design parameter and can be adjusted according to the code rate deployed at the transmitters [6], [8]. How to set $\alpha$ is beyond the scope of this paper, and hence, will not be considered any further. We simply assume that $\alpha$ is set to be larger than the minimum SNR required for the AP to decode the transmitted data correctly.

\section{B. Power adjustment}

For power-adjusted random access, the AP is made to receive all packets with the same receive power. However, all the users in a cell are positioned at different locations and may be subject to different channel conditions. Each user should therefore adjust his transmit power so that the AP's receive power from the users' transmissions may lie in the same range. We obtain the minimal receive power $\mathcal{R}^{*}=\mathcal{N} \alpha$ by the definition of SNR when there are no other simultaneouslytransmitting users. Therefore, we make each user adjust his transmit power autonomously to make the AP's receive power of his transmission not smaller than $\mathcal{R}^{*}$. Suppose there are $N$ transmit power levels, $\mathcal{T}^{1}, \cdots, \mathcal{T}^{N}\left(\mathcal{T}^{1}<\cdots<\mathcal{T}^{N}\right)$. We assume that the AP can always decode a transmitted signal within a target bit error rate by any subset of $N$ levels. To meet $\mathcal{R}^{*}$, the transmit power can be increased in a way $a$ priori agreed upon, as discussed in the next section.

The suitable transmit power level is referred to as the reference power level, which is used by most users called reference-power users. However, some users called excesspower users will use more power than their own reference power, and some users called deficient-power users attempt to transmit at a lower level than the reference power level. If the reference power level is $n^{*}$, the excess-power users transmit packets at levels $n^{*}+1, \cdots, N$. The users using $i$ levels higher power than their reference power are called $i$-excesspower users. For analytical simplicity, we suppose that access attempts of reference-power users do not cause collision to any excess-power users, and, similarly, $i$-excess-power users do not cause collision to $(i+1)$-excess power users. These assumptions hold mostly by the capture effect, although they might not hold with a slight probability when there are more than one simultaneously-transmitting user of smaller $i$.

\section{Power-Adjusted RANDOM ACCESS}

If every user maintains his transmit power such that the AP's receive power of his transmission is not smaller than $\mathcal{R}^{*}$, then a new user's initial access attempt may fail due to his collision with other users' attempts or use of insufficient transmit power. Collisions occur if there are other simultaneously-transmitting users. We attribute the failure to use of insufficient transmit power if the new user's data is received at a power level lower than $\mathcal{R}^{*}$. Occasionally, a user's attempt with a proper transmit power may fail when other simultaneously-transmitting users use excessive transmit power. We regard this case as collision, not the problem of using insufficient transmit power.

\section{A. Cause-of-Failure (CoF) estimation}

To prescribe a correct action after each transmission failure, we propose a probabilistic approach for determining the Cause-of-Failure (CoF). First, we define $p_{c}$ and $p_{l}$ to represent the probabilities of a random-access failure due to collision and use of insufficient transmit power, respectively. The failure caused by collision means that the transmit power used was sufficient. In other words, collision can occur only when the transmit power is sufficient. So, we can decide on the CoF by comparing $p_{c} \cdot\left(1-p_{l}\right)$ and a given threshold $\epsilon$.

If $p_{c} \cdot\left(1-p_{l}\right) \geq \epsilon$, the user can retry the failed transmission with the same transmit power. Otherwise (i.e., $p_{c} \cdot\left(1-p_{l}\right)<\epsilon$ ), the user can retry the transmission with more power, assuming that the failure was due to use of insufficient transmit power. The design parameter, $\epsilon$, lies in $[0,1]$ and determines the tendency of power increase. As $\epsilon \rightarrow 0$, collision becomes a dominant $\mathrm{CoF}$, while low transmit power becomes a dominant $\mathrm{CoF}$ as $\epsilon \rightarrow 1$. Note that the case of $\epsilon=1$ represents the conventional power-ramping algorithm; a transmission failure is always accompanied by a power increase at the next attempt.

Let $\Psi(n)$ be the probability that the required transmit power level is $n$ within a cell. We simply let $\sum_{i=1}^{N} \Psi(i)=1$. According to the distribution of users in a cell, the AP can 
obtain $\Psi(n)$ for the coverage area that requires the transmit power level $\mathcal{T}^{n}$.

Let $m$ and $n(n=1, \cdots, N)$ be the number of consecutive unsuccessful transmissions and the index of transmit power level, respectively. We can then calculate $p_{c}$ and $p_{l}$ as follows: ${ }^{1}$

$$
\begin{aligned}
p_{c}(m) & =(1-\exp (-\widetilde{G}))^{m}, m \geq 1 \\
p_{l}(n) & =\operatorname{Pr}\left(\mathcal{R}<\mathcal{R}^{*} \mid \mathcal{T}^{n}\right) \\
& = \begin{cases}\sum_{i=n+1}^{N} \Psi(i), & 1 \leq n \leq N-1, \\
0, & n=N\end{cases}
\end{aligned}
$$

where $\widetilde{G}$ is the measured effective load, and $p_{c}(m)$ is derived by the collision probability of slotted Aloha when the other users attempt to transmit packets at the same time. Clearly, $p_{c}$ will decrease with the increase of retransmissions, and $p_{l}$ will decrease with the increase of power level.

Let $\phi_{n}$ be the number of consecutive transmission attempts with the power level $\mathcal{T}^{n}$ until the user increases his transmit power. Then,

$$
\phi_{n}=\arg \min \left\{m: p_{c}(m) \cdot\left(1-p_{l}(n)\right)<\epsilon\right\},
$$

where $\phi_{n}$ increases as $n$ increases and $p_{l}(n)$ decreases. Note that $p_{l}(N)=0$; in other words, failures are always caused by collisions at the highest transmit power under our assumption, but the number of transmission attempts is bounded by some maximum for real applications. Usually, packets will be dropped after exhausting the maximum allowed number of attempts (using the final power level). Throughout this paper, we assume that failures can be recovered by an unlimited number of retransmissions at the maximum power level (i.e., $\left.\phi_{N} \rightarrow \infty\right)$ for analytical simplicity.

\section{B. Cause-of-Success (CoS) estimation}

When an initial random access was successful, the subsequent random access attempts can use the same transmit power instead of triggering the start of $\mathrm{CoF}$ algorithm from the lowest power level. If the channel condition remains unchanged, the user may reuse the latest-used (successful) power level for transmitting subsequent packets. However, the user's channel condition may change with time and, according to the $\mathrm{CoF}$ algorithm, with a slight probability, the power level may be increased even in case of successive collisions. If the present transmit power is too high for these reasons, causing the excessive receive power, it should be decreased. In our system model, however, the user doesn't know the channel condition for his next transmission. One possible way to handle this difficulty is to use the latest-used power level, and based on the result of using that power level, decide whether to change or retain the transmit power level.

We propose an algorithm to probabilistically determine the Cause-of-Success (CoS). While the $\mathrm{CoF}$ algorithm decides on the cause of failure after an unsuccessful random access attempt, the $\mathrm{CoS}$ algorithm decides on the cause of success after a successful random access. The $\mathrm{CoS}$ algorithm operates as follows. We define $p_{s}$ and $p_{h}$ as the probabilities of successful transmission without collision and at a power level causing the transmit power to be higher than the required

\footnotetext{
${ }^{1}$ We omit time and user indices in these parameters for notational simplicity.
}

level, respectively. If the success was due to collision-freedom, not high transmit power, the user can maintain his current power level. Thus, if $p_{s} \cdot\left(1-p_{h}\right) \geq \delta$, the user retains the present power level for the next random access. Otherwise (i.e., $p_{s} \cdot\left(1-p_{h}\right)<\delta$ ), the user makes the next random access attempt with a decreased power level. $\delta$ is also a design parameter that determines the trend of power decrease. Like $\epsilon$, a user tends to decrease the present power level if $\delta \rightarrow 1$, and maintains it if $\delta \rightarrow 0$.

Let $m$ and $n$ be the number of consecutive successful transmissions and the index of present power level, respectively. We then obtain $p_{s}$ and $p_{h}$ as:

$$
\begin{aligned}
& p_{s}(m)=(\exp (-\widetilde{G}))^{m}, m \geq 1 \\
& p_{h}(n)= \begin{cases}1-p_{l}(n-1)=\sum_{i=1}^{n-1} \Psi(i), & 2 \leq n \leq N, \\
0, & n=1\end{cases}
\end{aligned}
$$

where $p_{s}(m)$ is derived by the probability of no collision in slotted Aloha when no other users attempt to transmit.

Clearly, $p_{s}(m)$ decreases with the increase of $m$. Hence, we calculate the number, $\varphi$, of consecutive successful transmissions until the user decreases his transmit power as

$$
\varphi_{n}=\arg \min \left\{m: p_{s}(m) \cdot\left(1-p_{h}(n)\right)<\delta\right\} .
$$

Note that $\varphi_{1}$ is a trivial case since it already uses the lowest power level.

\section{Auto power fallback}

We now describe how $\mathrm{CoF}$ and $\mathrm{CoS}$ algorithms operate in a real setting. Each user terminal can realize these algorithms in a distributed fashion if users can measure, or be informed of, $\widetilde{G}$ and $\Psi(n)$ 's in order to calculate $p_{c}, p_{l}, p_{s}$, and $p_{h}$. Since this is impractical and/or inefficient, we develop instead a feasible mechanism for APs to execute.

At each $\mathrm{AP}, p_{c}$ and $p_{s}$ are computed by estimating the traffic load $\widetilde{G}$ within its cell, and $p_{l}$ and $p_{h}$ are computed by estimating $\Psi(n)$ 's from statistical users distribution and channel environment for given $N$ power levels. The AP then computes $\phi_{n}$ 's and $\varphi_{n}$ 's from Eqs. (4) and (7), respectively, and broadcasts the two sets every superframe via a common control channel or a beacon. Then, each user is informed of the number of consecutive successes or failures allowed until he decreases or increases his transmit power at each level.

This operation is akin to the auto rate fallback that is used for link adaptation in the IEEE 802.11 [14], and called auto power fallback (APF). Like in the auto rate fallback, APF can fix $\phi$ and $\varphi$ by assigning $\epsilon\left(1-p_{l}\right)$ and $\delta\left(1-p_{h}\right)$ instead of $\epsilon$ and $\delta$, respectively, thereby making the number of successive attempts irrelevant to the power level. This special case yields absolute fairness with respect to the successful access probability, and facilitates the implementation of our algorithm as there is no need to estimate $\Psi(n)$ 's.

\section{Performance Analysis}

APF drives every user to probabilistically and autonomously adjust his own transmit power to an appropriate level. According to our definition in Section II-B, those using a suitable transmit power level are called reference-power users. The traffic load of reference-power users is denoted by $G^{(0)}$. By 


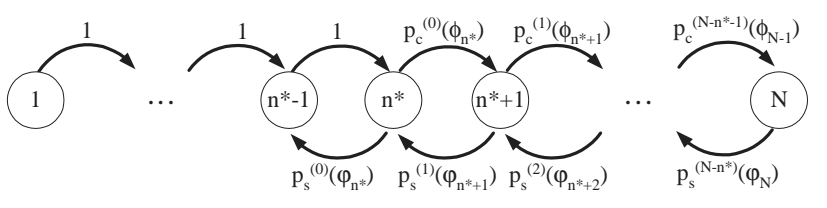

Fig. 1. The Markov chain model.

the probabilistic nature of the APF algorithm, some users called excess-power users will use more power than their reference power, because users increase their transmit power after making $\phi_{n}$ unsuccessful transmission attempts even if the transmission failures had really been caused by collisions. We denote the traffic load of $i$-excess-power users by $G^{(i)}$. On the other hand, some users called deficient-power users attempt to transmit at a lower level than their reference power level $n^{*}$ as a result of the CoS algorithm. We denote their traffic load by $G^{(-1)}$.

According to the assumption in Section II-B, $G^{(i)}$ dominates the others with the indices smaller than $i$ by the capture effect. In contrast, the users of $G^{(i)}$ cause collisions to the others with the indices not larger than $i$ for $i=0, \cdots, N-1$. Then, $p_{s}^{(i)}\left(\varphi_{n^{*}+i}\right)$ and $p_{c}^{(i)}\left(\phi_{n^{*}+i}\right)$, representing the $i$-excesspower users' probabilities of successes and collisions with the reference power level $n^{*}$ are given, respectively, by

$$
\begin{aligned}
& p_{s}^{(i)}\left(\varphi_{n^{*}+i}\right)=\left[\exp \left(-\sum_{j=i}^{N} G^{(j)}\right)\right]^{\varphi_{n^{*}+i}}, 1 \leq i \leq N-n^{*} \\
& p_{c}^{(i)}\left(\phi_{n^{*}+i}\right)=\left[1-\exp \left(-\sum_{j=i}^{N} G^{(j)}\right)\right]^{\phi_{n^{*}+i}}, 1 \leq i \leq N-n^{*}-1 .
\end{aligned}
$$

Meanwhile, access attempts by deficient-power users can cause collision to reference-power users by the definition of $\mathcal{R}^{*}$, so the probabilities of successes and collisions of reference-power users, $p_{s}^{(0)}\left(\varphi_{n^{*}}\right)$ and $p_{c}^{(0)}\left(\phi_{n^{*}}\right)$, are given by

$$
\begin{aligned}
p_{s}^{(0)}\left(\varphi_{n^{*}}\right) & =[\exp (-G)]^{\varphi_{n^{*}}}, \\
p_{c}^{(0)}\left(\phi_{n^{*}}\right) & =[1-\exp (-G)]^{\phi_{n^{*}}} .
\end{aligned}
$$

Using these probabilities, we can build a discrete-time Markov chain where each state represents a transmit power level. Fig. 1 shows the state diagram of our APF algorithm, when the reference level is $n^{*}$. Let $P_{n^{*}}(i+1 \mid i)$ represent the probability of transitioning from state $i$ to state $i+1$ when the reference level is $n^{*}$, then the one-step state transition probabilities are

$$
\begin{aligned}
& P_{n^{*}}(i+1 \mid i)= \begin{cases}1, & 1 \leq i \leq n^{*}-1 \\
p_{c}^{\left(i-n^{*}\right)}\left(\phi_{i}\right), & n^{*} \leq i \leq N-1\end{cases} \\
& P_{n^{*}}(i-1 \mid i)= \begin{cases}0, & 2 \leq i \leq n^{*}-1 \\
p_{s}^{\left(i-n^{*}\right)}\left(\varphi_{i}\right), & n^{*} \leq i \leq N .\end{cases}
\end{aligned}
$$

We define $\Pi_{n^{*}}(-1), \Pi_{n^{*}}(0), \Pi_{n^{*}}(1), \cdots, \Pi_{n^{*}}\left(N-n^{*}\right)$ as the probabilities that a user's power level becomes $n^{*}-$ $1, n^{*}, n^{*}+1, \cdots, N$ in steady-state. As $\sum_{i=-1}^{N-n^{*}} \Pi_{n^{*}}(i)=1$ for any $n^{*}$, we can establish a local balance equation between any two states. Hence, for $i=-1,0, \cdots, N-n^{*}$, we obtain $\Pi_{n^{*}}(i)$ that is expressed by transition probabilities. by

Then, the traffic load by deficient-power users is expressed

$$
G^{(-1)}=G \cdot \sum_{j=2}^{N} \Psi(j) \cdot \Pi_{j}(-1) .
$$

Similarly, the traffic loads by reference-power and excesspower users are given by

$$
G^{(i)}=G \cdot \sum_{j=1}^{N-i} \Psi(j) \cdot \Pi_{j}(i), \quad i=0, \cdots, N-1,
$$

where $\sum_{i=-1}^{N-1} G^{(i)}=G$.

As $G^{(i)}$ is a function of $p_{s}$ 's and $p_{c}$ 's, and each $p_{s}$ and $p_{c}$ are derived from $G^{(i)}$ 's, it is impossible to calculate them directly, but we can derive them recursively by setting $G$ to the initial value of $G^{(0)}$ and 0 to the others.

From $G^{(i)}$ 's, we obtain the successful access probability in a cell, $\bar{P}_{\text {succ }}$, as:

$$
\bar{P}_{\text {succ }}=\frac{G^{(0)}}{G} \exp (-G)+\sum_{i=1}^{N-1} \frac{G^{(i)}}{G} \exp \left(-\sum_{j=i}^{N-1} G^{(j)}\right) .
$$

Let $P_{\text {succ }}\left(n^{*}\right)$ be the successful access probability of reference-level- $n^{*}$ users, which is given by

$$
P_{\text {succ }}\left(n^{*}\right)=\Pi_{n^{*}}(0) \exp (-G)+\sum_{i=1}^{N-n^{*}} \Pi_{n^{*}}(i) \exp \left(-\sum_{j=i}^{N-1} G^{(j)}\right) \text {. }
$$

We now examine the average number of transmission attempts, $\bar{\chi}_{n^{*}, S}$, and the average energy consumption in steadystate, $\overline{\mathcal{E}}_{n^{*}, S}$, for making random access attempts until it becomes successful when the reference power level is $n^{*}$. Let $\bar{\chi}_{n^{*}}^{(i)}$ indicate the average number of transmission attempts at $i$-excess-power level when the reference level is $n^{*}$, and the case of $i=0$ represents the average number of transmission attempts at the reference level. If the transmission is successful at the $j$-th attempt with a certain power level, then there must have been $(j-1)$ collisions with this power level. Then, $\bar{\chi}_{n^{*}}^{(i)}$ is expressed by

$\bar{\chi}_{n^{*}}^{(i)}=\sum_{j=1}^{\phi_{n^{*}+i}} j \cdot p_{c}^{(i)}(j-1) \cdot\left(1-p_{c}^{(i)}(1)\right),\left(i=0, \cdots, N-n^{*}\right)$.

Let $\mathcal{W}_{i}$ be the energy consumed for transmitting a random access packet with transmit power level $i$, then we obtain $\overline{\mathcal{E}}_{n^{*}, S}$ as:

$$
\begin{aligned}
\overline{\mathcal{E}}_{n^{*}, S}= & \Pi_{n^{*}-1}\left(\phi_{n^{*}-1} \mathcal{W}_{n^{*}-1}+\sum_{i=0}^{N-n^{*}} \bar{\chi}_{n^{*}}^{(i)} \mathcal{W}_{n^{*}+i} \prod_{j=n^{*}}^{n^{*}+i-1} p_{c}^{\left(j-n^{*}\right)}\left(\phi_{j}\right)\right) \\
& +\Pi_{n^{*}}\left(\sum_{i=0}^{N-n^{*}} \bar{\chi}_{n^{*}}^{(i)} \mathcal{W}_{n^{*}+i} \prod_{j=n^{*}}^{n^{*}+i-1} p_{c}^{\left(j-n^{*}\right)}\left(\phi_{j}\right)\right) \\
& +\Pi_{n^{*}+1}\left(\sum_{i=1}^{N-n^{*}} \bar{\chi}_{n^{*}}^{(i)} \mathcal{W}_{n^{*}+i} \prod_{j=n^{*}+1}^{n^{*}+i-1} p_{c}^{\left(j-n^{*}\right)}\left(\phi_{j}\right)\right) \\
& +\cdots+\Pi_{N} \bar{\chi}_{n^{*}}^{\left(N-n^{*}\right)} \mathcal{W}_{N},
\end{aligned}
$$




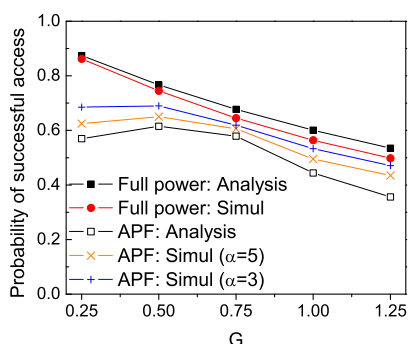

(a)

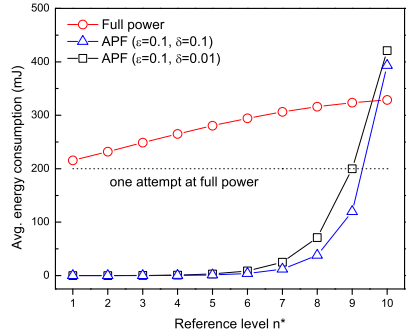

(b)
Fig. 2. (a) Probability of successful access as a function of $G$. It is compared with the simulation results; (b) Comparison of the average amount of energy consumption of full power and APF schemes (in steady-state, $G=0.75$ ).

where we define $\prod_{j=n^{*}}^{n^{*}-1} p_{c}^{\left(j-n^{*}\right)}\left(\phi_{j}\right)=\prod_{j=n^{*}+1}^{n} p_{c}^{\left(j-n^{*}\right)}\left(\phi_{j}\right)=$ 1 for notational simplicity. We omit the expression of $\bar{\chi}_{n^{*}, S}$ since it is an extraction of all $\mathcal{W}_{i}$ 's from Eq. (19).

\section{NumericAl RESUlts}

To evaluate the performance of APF, we use $\alpha=5 \mathrm{~dB}$ and the power levels ranging from $5 \mathrm{dBm}$ to $50 \mathrm{dBm}$, obtained with the path loss exponent equal to 4.0 and cell radius equal to $3 \mathrm{Km}$. To provide general performance figures regardless of cell characteristics throughout analytical results, we assume users' transmit powers are uniformly distributed over 10 levels when the transmit power is increased or decreased by $5 \mathrm{~dB}$ between 5 and $50 \mathrm{dBm}$. We also consider the Gaussian shadowing of zero mean and variance of $8 \mathrm{~dB}$. We set $\epsilon=0.1$ and $\delta=0.01$ by default.

To verify our analysis results, we conducted simulation, and performed each experiment 1,000,000 times using our simulator. We assume that 100 users are randomly distributed in a cell, generating packets with Poisson inter-arrivals. Since we use $5 \mathrm{~dB}$ as the difference between any two transmit-power levels, the simulation result of $\alpha=5 \mathrm{~dB}$ is closest to our analysis.

We plot the successful access probability as a function of $G$ in Fig. 2 (a) and compare the analytical and the simulation results. While our analysis assumed that $G^{(i)}$ does not cause collision to the others with the index larger than $i$ for $i=$ $0, \cdots, N-2$ in Eqs. (8) and (9), this does not hold in the simulation result as in a real case, when there are more than one user of the same $G^{(i)}$. However, it does not affect the performance significantly because of its low probability, so the simulation result at $\alpha=5$ is close to the analytical result. As the capture effect is more pronounced in the simulation from diverse receive powers, its successful access probability is higher than the analysis result. Especially, the probability increases with the decrease of $\alpha$, since it activates the capture effect more vigorously. Our algorithm is compared with the scheme that always transmits full power. Since the capture effect appears widely in this scheme, its overall successful access probability is greater than that of our scheme.

Fig. 2 (b) compares the average energy consumptions of APF and full transmit power when $G=0.75$. To calculate the amount of energy consumption without considering the effect of any other factor, we do not consider the energy consumed by other processing, and assume that the transmission duration for a random access packet is fixed at $2 \mathrm{msec}$. To obtain the amount of APF's energy consumption in steady-state, we use Eq. (19) assuming that transmissions are attempted as many times as necessary for the transmission to become successful at the final power level $N$. This assumption is also applied to the full-power scheme for a fair comparison. Consequently, at all but the final power level, the APF's energy consumption is much lower than the full-power scheme's, even though APF transits to different states. The result also demonstrates that APF still consumes less energy than the case of making only one transmission attempt with full power.

\section{CONCLUSION}

In this paper, we developed a new method called APF for power-adjusted random access. APF consists of $\mathrm{CoF}$ and $\mathrm{CoS}$ estimations, and probabilistically adjusts transmit power for random access. Since it is possible to control the sensitivity of changing the transmit power, APF can meet various objectives, such as energy-savings, successful access, and initial access. The numerical results confirm that APF finely tunes to a proper power level, thus making energy-savings in fixed and mobile wireless environments. It would be interesting to investigate the performance of exponential backoffs combined with APF and its application to multi-hop networks. Although APF's main focus is on WMANs or cellular networks, it will provide useful insights in solving the power-allocation problem for random access wireless networks. These are matters of our future inquiry.

\section{REFERENCES}

[1] N. Bambos, "Toward power-sensitive network architectures in wireless communications: Concepts, issues, and design aspects," IEEE Personal Commun. Mag., vol. 5, no. 3, pp. 50-59, June 1998.

[2] L. G. Roberts, "Aloha packet system with and without slots and capture," Comput. Commun. Rev., vol. 5, pp. 28-42, April 1975.

[3] Y. Yu, X. Cai, and G. B. Giannakis, "On the instability of slotted Aloha with capture," IEEE Trans. Wireless Commun., vol. 5, no. 2, pp. 257261, Feb. 2006.

[4] 3GPP TS 25.321 (V6.2.0), "Medium Access Control (MAC) protocol specification (Release 6)," Technical Specification Group Radio Access Network, June 2004.

[5] C. C. Lee, "Random signal levels for channel access in packet broadcast networks," IEEE J. Select. Areas Commun., vol. 5, no. 6, pp. 1026-1034, July 1987.

[6] R. O. LaMaire, A. Krishna, and M. Zorzi, "On the randomization of transmitter power levels to increase throughput in multiple access radio systems," Wireless Networks, vol. 4, no. 3, pp. 263-277, March 1998.

[7] G. del Angel and T. L. Fine, "Optimal power and retransmission control policies for random access systems," IEEE/ACM Trans. Networking, vol. 12, no. 6, pp. 1156-1166, Dec. 2004.

[8] G. del Angel and T. L. Fine, "Information capacity and power control for slotted Aloha random-access systems," IEEE Trans. Inform. Theory, vol. 51, no. 12, pp. 4074-4090, Dec. 2005.

[9] D. J. Goodman and A. A. M. Saleh, "The near/far effect in local Aloha radio communications," IEEE Trans. Vehi. Tech., vol. VT-36, no. 1, pp. 19-27, Feb. 1987.

[10] M. Zorzi and R. R. Rao, "Capture and retransmission control in mobile radio,” IEEE J. Select. Areas Commun., vol. 12, no. 8, pp. 1289-1298, Oct. 1994

[11] Y. E. Sagduyu and A. Ephremides, "Energy-efficient collision resolution in wireless ad-hoc networks," in Proc. IEEE INFOCOM 2003, San Francisco, CA, USA, March 2003.

[12] Y. Wu, et al. "Collision probability and throughput analysis in a powercontrolled DS-CDMA wireless network," IEEE Trans. Vehi. Tech., vol. 55, no. 1, pp. 350-359, Jan. 2006.

[13] Y.-J. Choi, S. Park, and S. Bahk, "Multichannel random access in OFDMA wireless networks," IEEE J. Select. Areas Commun., vol. 24, no. 3, pp. 603-613, March 2006.

[14] A. Kamerman and L. Monteban, "WaveLAN II: A high-performance wireless LAN for the unlicensed band," Bell Labs Technical Journal, pp. 118-133, Summer 1997. 